\title{
Cavity solitons in broad-area vertical-cavity surface-emitting lasers below threshold
}

\author{
Xavier Hachair, Stéphane Barland, Luca Furfaro, Massimo Giudici, Salvador Balle, ${ }^{*}$ and Jorge R. Tredicce \\ Institut Non-linéare de Nice, UMR 6618 Center National de la Recherche Scientifique-Université de Nice Sophia-Antipolis, \\ 06560 Valbonne, France \\ Massimo Brambilla, Tommaso Maggipinto, and Ida M. Perrini \\ INFM, Dipartimento di Fisica Interateneo, Università e Politecnico di Bari,Via Orabona 4, 70126 Bari, Italy
}

Giovanna Tissoni and Luigi Lugiato

INFM, Dipartimento di Scienze, Universitá dell'Insubria, Via Valleggio 11, 22100 Como, Italy

(Received 7 November 2003; published 21 April 2004)

\begin{abstract}
Cavity solitons are stationary self-organized bright intensity peaks which form over a homogeneous background in the section of broad area radiation beams. They are generated by shining a writing/erasing laser pulse into a nonlinear optical cavity, driven by a holding beam. The ability to control their location and their motion by introducing phase or amplitude gradients in the holding beam makes them interesting as mobile pixels for all-optical processing units. We show the generation of a number of cavity solitons in broad-area vertical cavity semiconductor microresonators electrically pumped above transparency but slightly below threshold. We analyze the switching process in details. The observed spots can be written, erased, and manipulated as independent objects, as predicted by the theoretical model. An especially tailored one is used to simulate the studied phenomena and to compare our simulations to the experimental findings with good agreement.
\end{abstract}

DOI: 10.1103/PhysRevA.69.043817

PACS number(s): 42.65.Sf, 42.70.Nq, 42.65.Tg

\section{INTRODUCTION}

The analysis of unstable and chaotic phenomena $[1,2]$ found a fertile ground in the field of nonlinear optics. In the late eighties, the main focus shifted from temporal effects to spatial pattern formation in the structure of the electromagnetic (e.m.) field in the transverse sections of broad-area radiation beams, when they interact with nonlinear media (see Refs. [3-5] and references quoted therein). The investigations in this domain offer an approach to parallel optical information processing, by encoding information in the transverse structure of the field. The idea is of considering the transverse planes as a blackboard on which light spots can be written and erased in any desired location and in a controlled way. Optical patterns may display an array of light spots, but are unsuitable for this task becausethe intensity peaks are strongly correlated with one another, so that they cannot be manipulated as independent objects. This task becomes possible, instead, using cavity solitons (CSs), a peculiar type of spatial solitons [6] which arise in a dissipative environment. They belong to the class of localized structures, which were discovered in other fields (see, e.g., Refs. [7,8] and for a general theory [9]) and arise under conditions of coexistence, in a nonlinear dynamical system, of a homogeneous stationary state and a patterned stationary state: for the same values of the parameters, according to the initial condition, the system may approach the homogeneous or the pattern state. Localized structures are intermediate between

\footnotetext{
*Also at Institut Mediterrani d'Estudis Avançats, IMEDEA (CSIC-Universitat de les Illes Balears), C/Miquel Marquès 21, E-07190 Esporles, Spain.
}

the two, in the sense that they coincide with the pattern state in a certain restricted region of the plane, and with the homogeneous state outside. By definition, localized structures must be independent of the boundary. A cavity soliton corresponds to a localized structure with a single peak. After pioneering works in the eighties [10-12], noteworthy attention was focussed on CSs since the midnineties [5,13-17]. They are generated in optical resonators containing nonlinear materials and driven by a broad area, coherent, and stationary holding beam (Fig. 1). The device is operated under parametric conditions such that the output is basically uniform over an extended region. However, by injecting a localized laser pulse one can write a CS where the pulse passes (at the location in the device cross section where the pulse impinges) and the CS persists after the pulse. The CSs written in this way can be erased by injecting again pulses in the locations where they lie; these pulses must be coherent and out of phase with respect to the holding beam [15]. Cavity solitons are not standard optical spatial solitons which arise from the balance of nonlinear self-focusing and diffraction or from nonlinear phase modulation, such as those considered in Ref. [6]. As a matter of fact, CSs may emerge even in

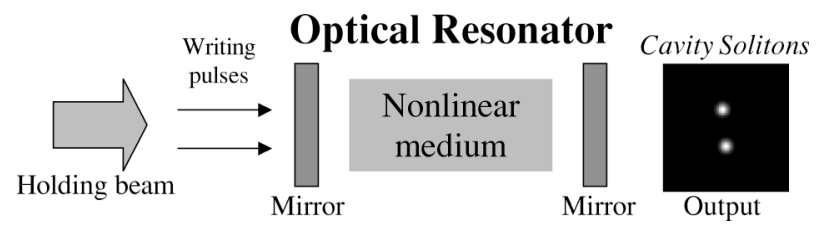

FIG. 1. A coherent, stationary, quasi-plane-wave holding field drives an optical cavity containing a nonlinear medium. The injection of narrow laser pulses creates persistent localized intensity peaks in the output (cavity solitons). 
presence of moderate self-defocusing, provided the carrier diffusion is large enough. They manifest a condition of bistability between an "on" localized state (induced by the writing beam) and an "off" low intensity state.

In presence of a phase (intensity) modulation in the holding beam, CSs tend to move to the nearest local maximum of the phase (intensity) profile [14]; for instance, by introducing a periodic phase modulation it is possible to create a reconfigurable array of CSs, which act as binary pixels. Experimental observations of localized structures in macroscopic cavities have been obtained [18-20]. Similar phenomena have been observed in systems with feedback [21-23] and recent works on control of large solitons arrays have been reported $[24,25]$. On the other hand, the observation of CSs in semiconductor microresonators is an important issue not only for fundamental physics but also for developing useful devices; theories for this configuration have been formulated in Refs. [26-31]. Optical patterns in semiconductor cavities have been observed in Refs. [32-36]. Phenomena of light localization, i.e., precursors or candidates for CSs, have been reported [33,37,38]; thermal effects play an important role in these observations. A clear-cut demonstration of objects that can be manipulated independently of each other and of the boundary, as it must be for CSs, has been obtained recently [39], using broad-area vertical cavity semiconductor laser, driven above transparency but slightly below threshold. A recent review on the topic of cavity solitons, with a rich list of references, can be found in Ref. [40]. In this paper we provide a more detailed description of some results briefly described in Ref. [39] and extend them. Especially, in the experimental part we demonstrate the generation of quite a number of CSs in the transverse section, whereas Ref. [39] reported on the writing/erasing of two solitons. In addition, we perform a careful analysis of the switching process of CSs. Furthermore, we provide a detailed description of the model used to simulate the experiment and present an extended comparison between the results of the numerical simulations and those of the experiment. These results arose from the close collaboration of the experimental group in Nice and the theoretical groups in Como and Bari. In Sec. II we present the experimental results, while in Sec. III we describe the theoretical and numerical results comparing them to the experimental evidences. The conclusions and perspectives are outlined in Sec. IV.

\section{THE EXPERIMENT}

\section{A. The experimental setup}

The laser where we are intended to generate the cavity solitons has been provided by Ulm university and is an oxidized bottom-emitter vertical-cavity surface-emitting laser (VCSEL) with Bragg mirrors consisting of 20.5 pairs on the bottom side and 30 pairs on the top side, and three quantum wells emitting around $970 \mathrm{~nm}$ at threshold [41]. Its diameter is $150 \mu \mathrm{m}$.

An external-cavity laser in Littrow configuration (Fig. 2) provides the holding beam (HB), which can be tuned in the range $960-980 \mathrm{~nm}$ by steps of $51 \mathrm{GHz}$ (which corresponds to the longitudinal mode separation of the laser) and, for

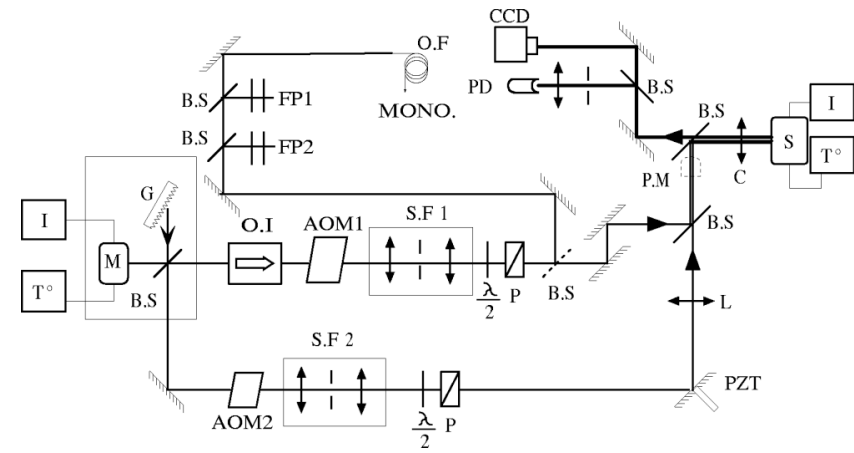

FIG. 2. Experimental setup. $M$, high power edge emitter laser; $I$, Current driver stabilized up to $0.01 \mathrm{~mA} ; T^{O}$, Temperature controller; $G$, grating; OI, optical diode; FP, Fabry-Perot resonators; AOM, Acousto-optic modulators; SF1, Beam expander-configurator with spatial filtering; SF2, Beam reducer configurator with spatial filtering; S, broad-area vertical cavity surface emitting laser; $C$, collimator; CCD, camera; PD, Photodetector; PZT, Piezoelectric ceramic; $\mathrm{M}$ and $\mathrm{BS}$, mirrors and beam splitters; PM, power meter (optional); $\lambda / 2, \lambda / 2$ wave plates; $P$, polarizes; $\mathrm{OF}$ and MONO, optical fiber and monochromator.

each step, it can be tuned continuously on a range of $6 \mathrm{GHz}$. The measured linewidth is less than $1 \mathrm{MHz}$ on long-time acquisitions (larger than $1 \mathrm{~min}$ ), while the maximum power attainable is $100 \mathrm{~mW}$. This beam is spatially filtered and it is prepared by an optical system in order to obtain a collimated beam having a waist of about $300 \mu \mathrm{m}$. This beam, whose intensity can be considered almost uniform across the whole section of the VCSEL, is injected into the cavity along the optical axis. An optical isolator (Gsänger modulator FR $500 / 1100$, return loss $>30 \mathrm{~dB}$ ) is placed at the beam exit of the external-cavity laser in order to avoid any disturbance from back reflections and from the slave output. The intensity of the injection beam entering in the VCSEL (before the collimator) can be adjusted up to $33 \mathrm{~mW}$. The holding beam power is controlled by an acousto-optic modulator (maximum suppression 30\%) together with a polarizer. The second output coming from the external cavity laser is used as writing beam (WB). This beam is prepared to obtain a waist of $10-15 \mu \mathrm{m}$ while its maximum injectable power in the VCSEL is $1 \mathrm{~mW}$. The writing beam power is controlled by an acousto-optic modulator (maximum suppression 30\%) and by a polarizer. Writing beam phase relationship with respect the $\mathrm{HB}$ is controlled by piezo positioning of a mirror on its own path.

The reflected output of the VCSEL is monitored by a charge-coupled device (CCD) camera Pulnix 765E, on which we form the image of the emitted near field. A photodetector Thorlabs D400 (less than 100 ps rise time) monitor a small portion of the VCSEL transverse plane in order to detect the switching dynamics of a CS. A digital oscilloscope Lecroy Wavemaster $8600 \mathrm{~A}(6 \mathrm{GHz}$ analog bandwidth, $20 \mathrm{GHz}$ digital bandwidth) is used for monitoring the detector output. For measuring the optical spectrum of the holding beam we used three kinds of instruments: a large free spectral range scanning Fabry-Perot interferometer (resolution $2 \mathrm{GHz}$, FSR $270 \mathrm{GHz}$ ), a high resolving Fabry Perot interferometer (resolution $300 \mathrm{MHz}$, FSR $30 \mathrm{GHz}$ ), and a monochromator with a 


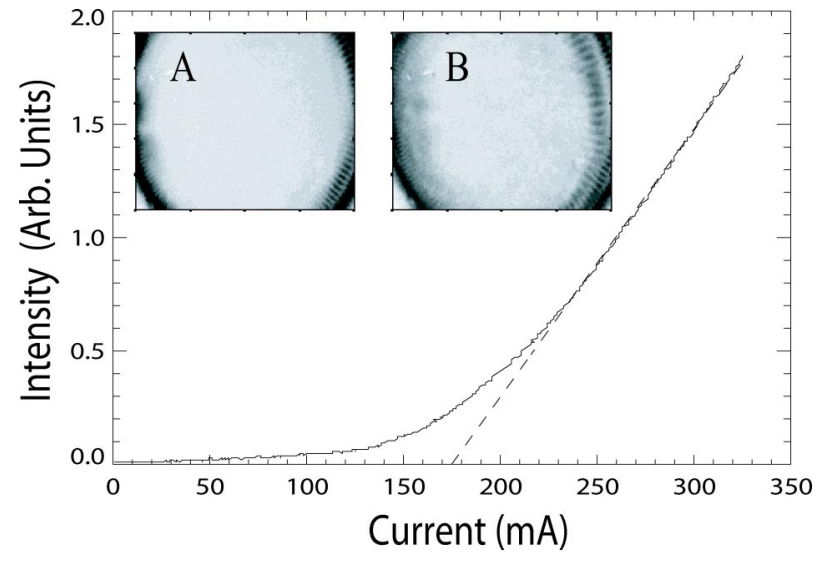

FIG. 3. Light intensity output vs pumping current for the solitary VCSEL. inset: (a) Average intensity profile at $J=200 \mathrm{~mA}$, (b) emission profile at $J=300 \mathrm{~mA}$.

resolution of $0.5 \AA$ for having absolute estimation of the injection wavelength. The same set of instruments have been used to measure also the VCSEL output spectra. A power meter can be inserted into the injections beam path just before the VCSEL in order to estimate the writing beam and holding beam power.

\section{B. The solitary laser}

The light intensity output of the solitary VCSEL as function of the pumping current $(J)$ is plotted in Fig. 3. Applying the conventional definition of laser threshold, we infer $J_{t h}$ $=175 \mathrm{~mA}$. In fact, this definition of laser threshold, where the intensity output is integrated through the whole transverse plane of the device, is not meaningful when dealing with such broad-area laser. Looking at the output profile at $J_{t h}$ [inset (a) of Fig. 3] it is evident that the emission occurs only around the boundaries of the device. This "ring" profile for $J=J_{t h}$ is due to the current crowding close to the insulating boundaries. As the pumping current is increased, the laser emission occurs progressively in the whole transverse plane, but no emission is detected monitoring the central part of the device up to $J=300 \mathrm{~mA}$ [inset (b) of Fig. 3]. In other words, in such a broad-area VCSEL, every point in the transverse section has an emission threshold depending on its radial distance from the center of the device. It is useful to monitor the local emission spectrum across a section of the VCSEL. Figure 4(a) shows clearly that for $J=300 \mathrm{~mA}$, there is emission at several well defined frequencies only near the boundary. For $J<300 \mathrm{~mA} 80 \%$ of the transverse section of the device does not emit. We will always operate at current values for which the central region is not emitting. Another important test in order to asses that we are operating the VCSEL as an amplifier (at least in the transverse region of interest) is to verify that, under injection of an external field, the VCSEL emission frequency is locked to the injection frequency. In Fig. 4(b) we show that up to $J=320 \mathrm{~mA}$ the central region is indeed locked to the frequency of the external field while the region close to the boundary shows emission at several frequencies.
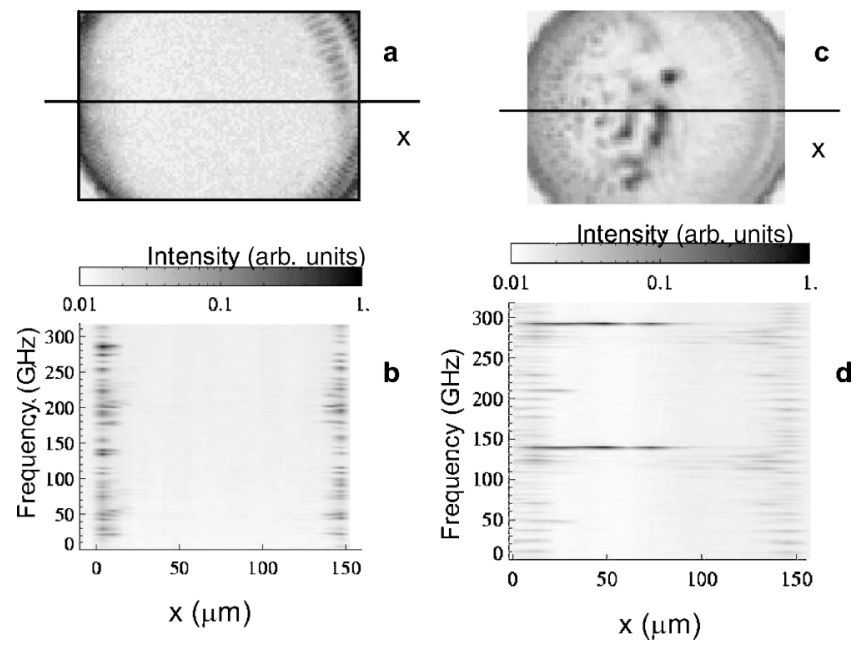

FIG. 4. Average intensity profile (top) and spatially resolved optical spectra across a section of the VCSEL (bottom) for two experimental situations: Free running laser pumped at $J=300 \mathrm{~mA}$ (left) and VCSEL with injected field $P_{h b}=8 \mathrm{~mW}$ at $J=320 \mathrm{~mA}$ (right). The darker lines (high intensity) correspond to the frequency of the injected field. The frequency interval between the two dark lines corresponds to the free spectral range of the Fabry-Perot resonator. No defined frequency is observed in the homogeneous region because the intensity is not high enough to allow a measurement with the sensitivity of our detectors.

\section{The broad-area VCSEL under injection of an external field}

The VCSEL output profile when we inject an external monochromatic field is shown in Fig. 5. In order to maximize the VCSEL gain we operate it at a temperature for which its cavity resonance frequency is close to the gain curve peak. The VCSEL amplification of an external field is maximum when the injected frequency is close to the cavity resonance of the VCSEL cavity. In this situation the output profile can be divided in two parts [Figs. $5(\mathrm{~b})-5(\mathrm{~g})]$ : a left part where a patterned structure is formed, and a right part where the reflected intensity is uniform and has a low intensity level. We have here to emphasize that this asymmetry is not due to misalignment of the injected beam which is parallel to the emission axis of the VCSEL. These two zones are separated by a vertical line and the pitch of the spatial structure uniformly decreases to the left of the device.

These emission profiles can be understood considering that broad-area VCSELs usually exhibit a strong gradient of
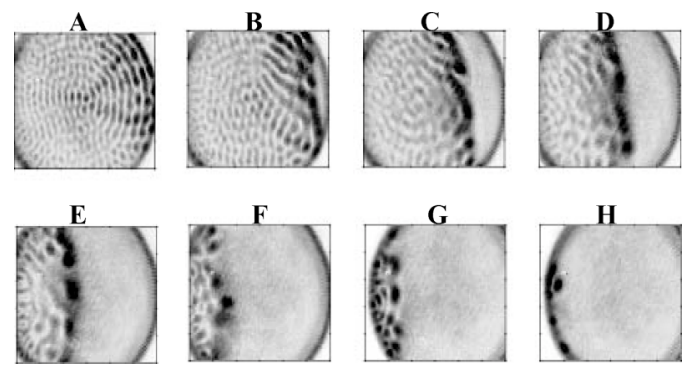

FIG. 5. Average intensity profiles of the VCSEL under different injection frequency around $970 \mathrm{~nm}$. From (a) to (h) the frequency is decreased by step of $50 \mathrm{GHz}, J=180 \mathrm{~mA}, P_{h b}=8 \mathrm{~mW}$. 

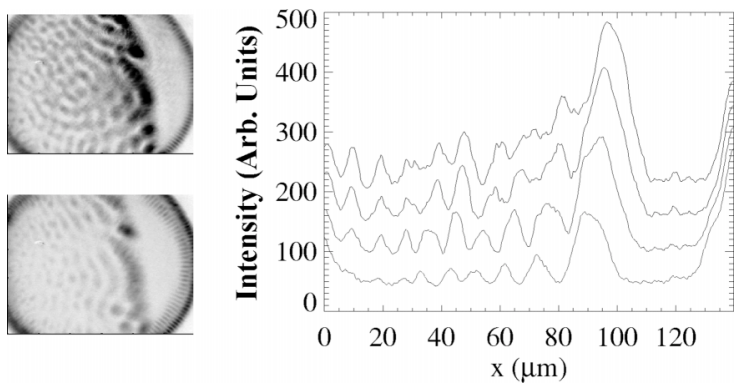

FIG. 6. Modulational instability border shifting as the injection intensity is increased, $J=180 \mathrm{~mA}, P_{h b}$ from $1 \mathrm{~mW}$ (lowest in the graph) to $8 \mathrm{~mW}$ (highest in graph). The different curves have been offset for clarity.

the cavity length along the transverse section due to the nonparallel layers forming the cavity, owing to the standard epitaxial growth techniques. By consequence, in our system, the cavity resonance varies along one direction of the transverse plane. The line separating the pattern and the homogeneous field phase can be interpreted as the locus of the spatial positions where the local values of the cavity resonance and field intensity meet the condition for the onset of a (pattern-inducing) modulational instability. We recall that in a homogeneous system, for all other parameters fixed, this boundary is defined in parameter space only by a critical detuning $\theta_{c}$ between the injected field frequency $\left(\omega_{i}\right)$ and the longitudinal cavity resonance $\left(\omega_{c}\right): \theta=\left(\omega_{c}-\omega_{i}\right) / \kappa$, being $\kappa$ the cavity half-width. For $|\theta|>\left|\theta_{c}\right|$ a pattern develops. Instead, in our system, due to the gradient of the cavity resonance, the modulational instability (MI) occurs in the transverse plane of the VCSEL and the instability boundary manifests itself as a separation between a patterned spatial region, and a uniform one. The numerical studies (see Sec. III) show that the critical detuning $\theta_{c}$ depends on the power of the injected field $\left(P_{h b}\right)$, as we show in Fig. 6. Varying the injected field frequency while keeping fixed its intensity, we observe a rigid shift of the whole pattern in the transverse plane (Fig. 5). This is due to the shift of the MI boundary to the new locus of points where the critical detuning condition is satisfied. This observation can be used in order to estimate the gradient of the cavity resonance in the VCSEL [42]. In this particular device, a cavity resonance gradient of $2.34 \mathrm{GHz} \mu \mathrm{m}^{-1}$ is measured along the horizontal dimension, which leads to a resonance frequency difference of $351 \mathrm{GHz}$ between one side and the other of the amplifier. This value is in excellent agreement with the value found from wafer measurements [43]. In Fig. 7 we show the near field output of another VCSEL sample where the wafer growth process has been improved in order to decrease the cavity resonance gradient to $0.27 \mathrm{GHz} \mu \mathrm{m}^{-1}$.

\section{The cavity solitons: control and parameter space}

According to theoretical predictions (see Sec. III) CSs are stable solution for a given range of values for the cavity detuning $|\theta|<\left|\theta_{c}\right|$. Then, they can be observed experimentally in the homogeneous region of the VCSEL under injection (see Fig. 7(c), for example). Moreover, the presence of
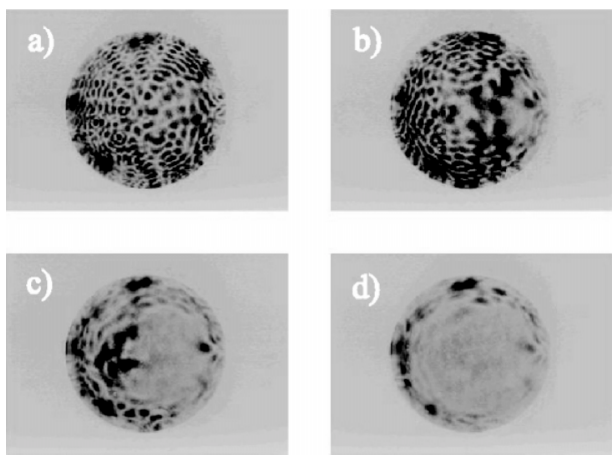

FIG. 7. Average intensity profiles of a second sample of VCSEL under different injection frequency around $970 \mathrm{~nm}$. From (a) to (d) the frequency is decreased by step of $50 \mathrm{GHz}, J=200 \mathrm{~mA}, P_{h b}$ $=8 \mathrm{~mW}$.

the cavity resonance gradient limits the existence of the CSs to a small region around the vertical line separating the patterned region from the homogeneous region. We choose the sample with the smallest cavity gradient, since we dispose of the largest portion of transverse plane fulfilling the criteria for stable CSs. We fix all parameter values as in Fig. 7(c) and we inject the writing beam into the homogeneous region. Starting with no spot, the WB is capable of generating a high intensity spot with a diameter on the order of $10 \mu \mathrm{m}$ when it is in phase with the holding beam. If we remove the WB, the bright spot remains on indefinitely. We then apply this beam in a different location without changing any parameter value and a second spot is generated without perturbing the first one we have created. This one will also persist after removal of the WB. We reach, then, the situation in which two spots exist. Changing the phase of the WB by $\pi$ in respect with the $\mathrm{HB}$ and reinjecting it successively at each location, where the spots have been created, we erase each of them in an independent way. The full series is displayed in Fig. 8 and we claim these spots being CSs [39]. The minimum power requirement $P_{w b, \min }$ for the WB in order to switch on a CS depends on $P_{h b}, \theta$, and $J$. For measuring $P_{w b, \min }$ we fix $\theta$ like in Fig. 7(c). We find that for $J=262 \mathrm{~mA}, P_{w b, \min }$ ranges from 1 to $20 \mu \mathrm{W}$ for $P_{h b}$ ranging, respectively, from $8 \mathrm{~mW}$ to $1 \mathrm{~mW}$, while for $J=252 \mathrm{~mA}, P_{w b, \min }$ ranges from 1 to $20 \mu \mathrm{W}$ for $P_{h b}$ ranging, respectively, from $27 \mathrm{~mW}$ to $15 \mathrm{~mW}$.

It is interesting to measure the rise time of the CSs after application of the WB. In Fig. 9 we show the time series displaying the rising front of the CS. The rise time of the CS is $570 \pm 50 \mathrm{ps}$. This value is not significantly affected by parameters variations, provided that the CS can be switched on. It is important to remark that the time measured is the build-up time of the CS intensity but we cannot claim that this is the total CS switch-on time after application of the WB. Measurements for quantifying a time-delay between the application of the WB and the onset of the CS intensity buildup are in progress.

Cavity solitons may be switched on by means of the writing beam but they can also appear spontaneously induced by the noise present into the system when the holding beam power is close to the critical value for which the homoge- 

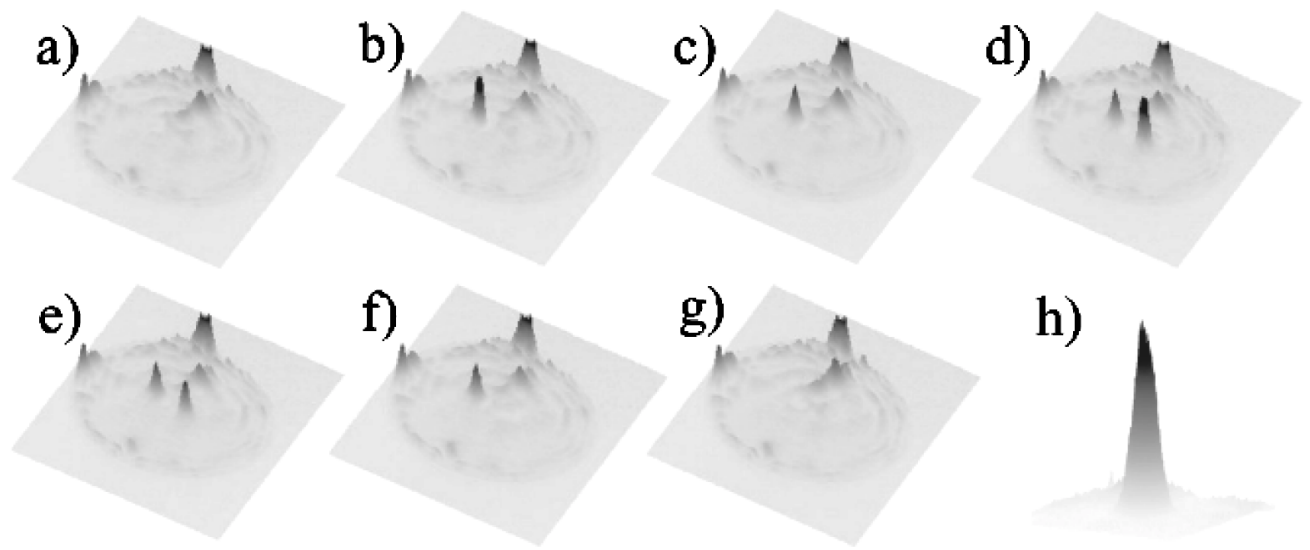

FIG. 8. Intensity distribution of the output field. The holding beam is always on, and all parameters are kept constant: $P_{h b}=10 \mathrm{~mW}, J$ $=262 \mathrm{~mA}$. (a) The writing beam (WB) is blocked; (b) a $15 \mu \mathrm{m}$ focused WB $\left(P_{w b}=8 \mu \mathrm{W}\right)$ targets a point into the homogeneous region; it induces the appearance of a single CS; (c) the WB is blocked again, the CS remains; (d) the WB is displaced in position and switched-on again and it generates a second CS; (e) the WB is blocked again and the two bright spots coexist; (f) the WB targets again the second CS, but the relative phase of WB with respect to HB has been changed of $\pi$ and the CS is erased; $(\mathrm{g})$ the WB targets again the first CS, but the relative phase of WB with respect to $\mathrm{HB}$ has been changed of $\pi$ and even the first CS is erased. Once the WB is blocked, the intensity distribution is identical to (a). In (h) we plot the CS profile.

neous solution is not stable anymore [this value will be illustrated and commented in Sec. III (see Fig. 15)]. They also appear decreasing the holding beam power from values where only pattern solutions are stable to values where only CSs and the homogeneous solution are stable. In Fig. 10 we show experimentally, as function of the VCSEL bias, the values of the holding beam power at which, for increasing power, the homogeneous solution (Fig. 10, inset 1) looses its stability and pattern (Fig. 10, inset 3) develops (Fig. 10, upward arrow). For decreasing $\mathrm{HB}$ power, patterns evolves to CSs (Fig. 10, inset 2). Continuing to decrease the HB power, at a critical value the CS solution looses its stability and the system switch to the homogeneous solution (Fig. 10, downward arrow). These transitions, as always in real systems close to bifurcation, are inevitably smoothed by the presence of noise. Therefore, close to the parameter values where the

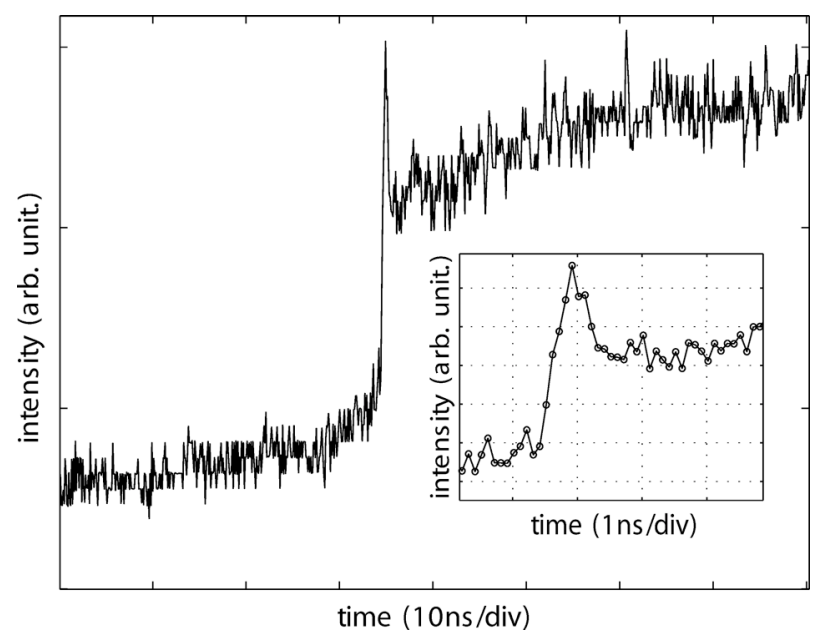

FIG. 9. Measure of the rise time of the CS and (inset) zoom onto a smaller time window. $P_{h b}=3 \mathrm{~mW}, P_{w b}=10 \mu \mathrm{W}$. homogeneous solution or CS solution becomes unstable, the noise may induce spontaneous jumps of the system towards the other solution. This results in a relative uncertainty of parameter values of about $20 \%$ for the borders of the bistability region (gray part in Fig. 10) where CSs coexist with homogeneous solution.

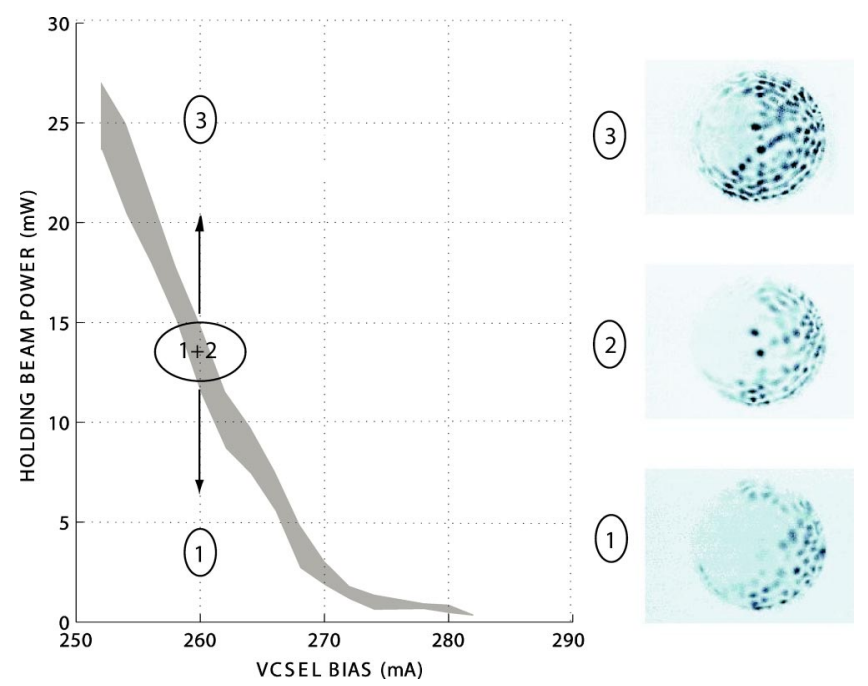

FIG. 10. CSs existence domain as a function of the parameters. Upward arrow indicates the border in the parameter space where the homogeneous solution switches off for increasing HB intensity. Downward arrow indicates the border in the parameter space where the CSs switches off for decreasing HB intensity. Region (1) is characterized by the output profile of inset (1), region (3) is characterized by the output profile of inset (3) and in the gray region $(1+2)$ CSs (inset 2) and homogeneous solution coexist. Note that the profiles are flipped horizontally with respect the ones shown in Figs. 5 and 7. 


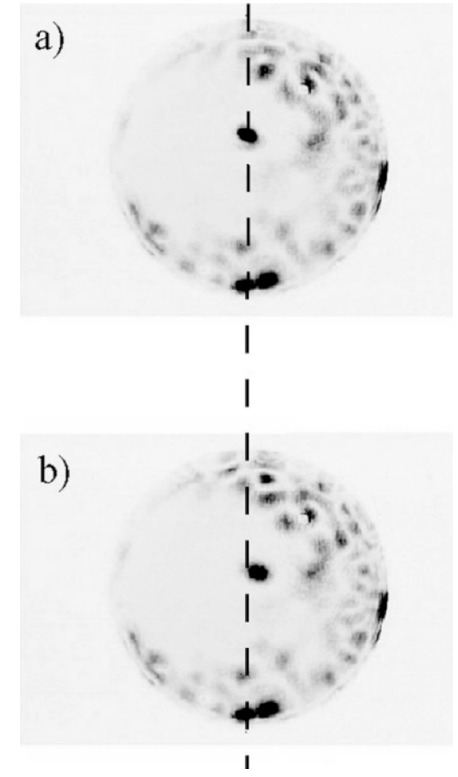

FIG. 11. CS drift: we target an arbitrary point with the WB and, as we remove it, CS migrates in a point nearby. The vertical line helps as reference for the CS position. Note that the profiles are flipped horizontally with respect the ones shown in Figs. 5 and 7.

\section{E. The cavity solitons: positioning}

By changing the frequency of the injected field we find various positions where a CS can be located, always in the homogeneous emission region and close to the border with the pattern region. Thus, such structures exist in a relatively wide range of injected field intensity and/or pumping current and always close to the critical detuning value corresponding to the modulational instability. Anyway, we are not able to create a CS in an arbitrary position inside the transverse domain where they are possibly stable. In Fig. 11 we target with the WB a point in a slightly different location with the one targeted in Fig. 8(b). We can generate a CS but after removal of the writing beam it migrates towards the position of Fig. 8(b), suggesting that this location is an attracting locus for CS. Two elements determine this behavior. First the presence of a roughness of the layer as well as random distributed impurities across the transverse plane of the device which trap the CS. Second the cavity resonance gradient which acts as "wind" and tends to blow the CS towards the pattern region with a velocity proportional to the gradient.

We have measured the speed of the CS. The CS drifts of $18 \mu \mathrm{m}$ in $38 \mathrm{~ns}$ which means a speed value of $470 \mathrm{~m} / \mathrm{s}$. This value is in good agreement with the value predicted by the theory for the amount of gradient present in our device (see Sec. III D). Moreover, the theory explains also the trapping role of the sample roughness. The conditions for such "trapping" obviously depends on the balance among the local resonance and the large-scale resonance cavity gradient. In conclusion, the presence of the cavity gradient induces a drift of the CSs and limits their existence to a small region in the transverse plane. A strong effort in solving this problems is in progress for the future fabrication of new samples. On the other side, the presence of impurities and the inevitable roughness of the layers trap the CSs. This enables the obser- a)

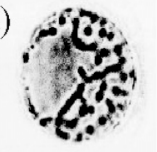

d)

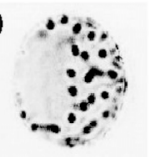

b)

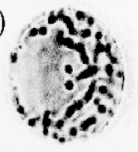

e)

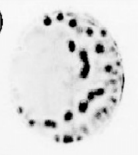

c)

f)

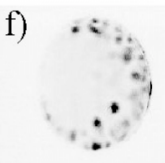

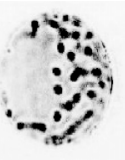

FIG. 12. Intensity distribution of the output field for decreasing HB power, $J=280 \mathrm{~mA}$. From (a) to (f) the holding beam power is scanned downward from $33 \mathrm{~mW}$ to $2 \mathrm{~mW}$. The HB has been tilted in order to vectorially compensate the force exerted by the cavity length. Note that the profiles are flipped horizontally with respect the ones shown in Figs. 5 and 7.

vation on averaging CCD camera possible but it makes difficult an arbitrary positioning of the CSs across the device.

Alternatively, we can observe CSs if we vectorially compensate the force exerted by the cavity length gradient with a force exerted by an opposite gradient. Such antagonistic gradient could be easily introduced experimentally in the intensity or in the phase of the holding beam. An easy way to introduce a phase grating in the holding beam is by injecting a holding beam tilted witch respect the VCSEL cavity axis. A beam tilted in the plane defined by direction of the cavity resonance gradient and the axis of the VCSEL cavity results in a phase gradient which may compensate the cavity resonance gradient if the angle of tilting is chosen with the right value. In Fig. 12 we have used an holding beam tilted in order to compensate the cavity gradient. Starting from maximum value of $P_{h b}$ and decreasing it we observe, in agreement with theoretical results, "stripe like" patterns that contracts to leave CSs, confirming the conceptual interpretation of CSs as "remains of bifurcating pattern." Choosing properly the parameters we are able to create up to seven CSs (Fig. 13) almost filling the region where they are stable. This result is strongly encouraging for the future in order to develop gradient-free broad area VCSEL. As further step, once these devices are obtaired, it will be very interesting to analyze the interaction between them as a function of their distance and to implement the all-optical reconfiguration of CSs array.

\section{THEORY AND SIMULATIONS}

\section{A. The model}

We described the field dynamics of the device studied in the experiment performing tailored simulations in a model

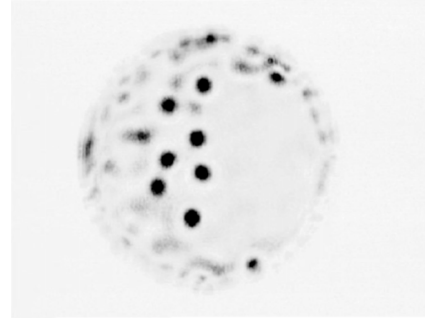

FIG. 13. Intensity distribution of the output field showing the presence of seven CSs. $P_{h b}=25 \mathrm{~mW}, J=290 \mathrm{~mA}$. 
where we first predicted existence of CSs [26,28]. The basic dynamical equations for the slowly varying coherent field and carrier density, in the paraxial and mean field limit approximations, read

$$
\begin{gathered}
\frac{\partial E}{\partial t}=-\kappa\left[(1+i \theta) E-E_{I}-i \Sigma \chi(N) E-i a \nabla_{\perp}^{2} E\right], \\
\frac{\partial N}{\partial t}=-\gamma\left[N-\operatorname{Im}[\chi(N)]|E|^{2}-I-d \nabla_{\perp}^{2} N\right],
\end{gathered}
$$

where $E, N$ are the normalized electric field and the carrier density (normalized to its transparency value), $\kappa$ is the cavity decay rate, $\gamma$ is the nonradiative recombination rate, $\theta$ is the cavity detuning parameter, $E_{I}$ is the normalized input field, $I$ is the normalized current profile, $\Sigma$ is the cooperativity parameter, $\chi$ is the susceptibility of the material, and $\nabla_{\perp}^{2}$ is the transverse Laplacian where $a$ and $d$ are the diffraction and diffusion coefficients, respectively. A more detailed discussion of the parameter definitions and of the scalings can be found in Ref. [15].

In recent times the above model was refined to include a first-principle description of the semiconductor susceptibility, many-body effects, and a few other relevant corrections $[29,30]$, moreover fundamental quantum-based models of semiconductor response have been successfully proposed $[31,44]$. As it turns out, the approximation to the semiconductor susceptivity

$$
\chi(N)=-(\alpha+i)(N-1)
$$

yields results which are in excellent agreement with the experiment and already proved to provide results (MI thresholds, pattern variety, CS properties and dynamics) qualitatively similar to more refined models (e.g., compare Refs. $[28,29])$. In Eq. (3), $\alpha$ is the linewidth enhancement factor [45]. The peculiarity of the experimental observations resides mainly in the particular shape of the sample, the variation of the microcavity resonance across the VCSEL's diameter, the current crowding on its outer border and-as our simulations evidenced-the fluctuations in the resonance transverse profile. This required us to introduce phenomenological modifications, increasing the complexity of our simulations and preventing an analytical explanation of the emerging pattern variety.

These modifications are summarized as follows.

(1) A broad Gaussian profile for the holding beam, to reproduce its experimental shape, $E_{I}(x, y)=E_{I} \exp \left[-\left(x^{2}\right.\right.$ $\left.\left.+y^{2}\right) / 2 \Sigma^{2}\right]$, where the origin of the $(x, y)$ coordinate system is chosen at the center of the integration grid and $\Sigma$ is a broad width (usually twice the spatial size of the integration grid) so that $E_{I}(x, y)$ is almost plane wave where the injected current is not zero (see next point 4 ).

(2) A constant gradient in the cavity length, which amounts to substituting the parameter $\theta$ in Eq. (1) with a spatially dependent detuning

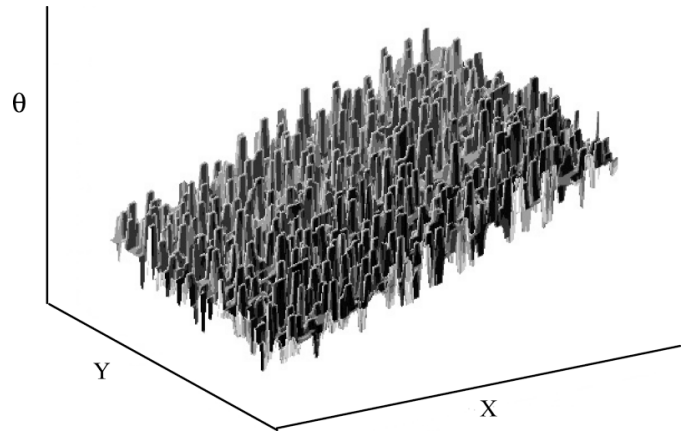

FIG. 14. The transverse spatial profile of the cavity resonance $\theta(x, y)$. A spatially distributed stochastic process was added to simulate the roughness induced by the layer jumps in the epitaxial deposition of the DBRs. The deterministic profile of the resonance shows a linear gradient, as measured in the experiment.

$$
\theta(x, y)=\theta_{1}-\eta x,
$$

where $x$ is the horizontal coordinate. This accounts for the measured varying cavity resonance (see Sec. II C); the chosen values for $\eta$ were induced from the experimental indications about the distributed Bragg reflector (DBR) reflectivity and the cavity frequency variations along the sample diameter.

(3) The irregularities in the layers of the Bragg reflectors, which are modeled by setting $\theta_{1}=\theta_{0}+\delta \theta(x, y)$, where $\delta \theta(x, y)$ is a Gaussian stochastic process with zero average and transverse correlation length of a few microns. The magnitude of the fluctuations depends on the reflectivity and on the distribution of layer jumps associated to the epitaxial deposition. The correlation length is related with the typical transverse dimensions of the layer defects. Values for this stochastic process were induced from previous works [34]. A stochastic realization of $\theta(x, y)$ is shown in Fig. 14 .

(4) A spatial profile of the electric current $I(x, y)$ capable to simulate two basic features of the sample under observation. One is the circular symmetry of the sample, which is related to the physical etching of the VCSEL and to the annular electrode deposition: in this respect we assume that the current is zero outside a circle and this is sufficient to qualitatively reproduce the occurring patterns. The other is the current crowding observed on the outer border of the sample, due to the annular contact. Since the investigated sample was a bottom emitter, a particular architecture specifically cued to make the current-density distribution more homogeneous inside the sample, it turns out that a top-hat profile is enough to adequately describe the patterns and the CSs in the operative regimes reported in Sec. II, while an annular crowding was sufficient to qualitatively match the process of emission close to threshold and few other details.

\section{B. Pattern variety and symmetries}

The general study of the MIs and pattern formation in an infinitely extended system has been provided in Ref. [28], further refinements and extensions were provided in Refs. $[46,47]$; here we provide or recall results for a specific para- 


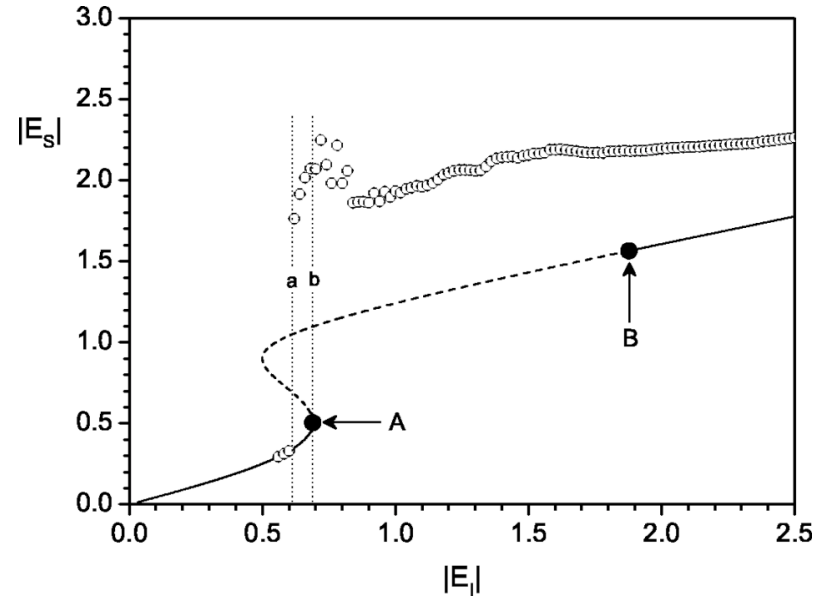

FIG. 15. S-shaped input-output curve for the homogenous stationary solution of Eqs. (1) and (2). The broken part of the curve shows the unstable region where, due to a MI, the system can be expected to realize a patterned profile. Points $A$ and $B$ mark the (lower/higher, respectively) thresholds of the instability and are calculated by performing a linear stability analysis on an infinitely extended medium along Ref. [28]. The value of the input field at points $A$ and $B$ is 0.69 and 1.88 , respectively. The two vertical lines $a$ and $b$ delimit the region where patterns and stable homogeneous solutions coexist. Circles refer to solutions obtained by direct integration of Eqs. (1) and (2) for a circular current profile; on the left of line $a$, they correspond to homogeneous solution, while on the right they plot the maxima of patterned states. Other parameters are: $d=0.052, \theta=-2.25, \Sigma=0.90, \alpha=5.0$.

metric set suitable for comparison with the device and setup adopted in the experiment.

Figure 15 shows the homogenous steady state curve (Sshaped solid/broken line) of the emitted field versus the injected field, the broken part of which marks the part of the curve unstable versus spatial perturbations. The arrows indicate the lower and higher thresholds of the modulational instability, as predicted by the linear stability analysis. The hollow circles show the results of the simulations in a bounded sample; they mark the maxima of the patterns realized by the system when the homogeneous emission loses stability in favor of spatial modulations. Note the existence of a range of input field values, between the vertical lines $a$ and $b$, to the left of point $A$, the lower MI threshold (which proved to be rather close to that of a broad area VCSEL, despite the existence of boundaries imposed both by the holding field shape and the material/current confinement), where patterns coexist with a homogeneous background. This is the elective regime where CSs can be obtained. The wide superposition of patterns and homogeneous solutions to the right of the higher MI threshold $B$ is a feature due to the system's boundaries and will be described further on in the text. In this work, we specifically concentrate on features related to the sample structure and geometry: to this purpose our approach was to gradually introduce the modifications listed above in order to evidence their implications on the morphogenesis and gain a better comprehension of the experimental observations.

The first step was thus to study the role of the circularly symmetrical boundaries of the VCSEL: for example, it is

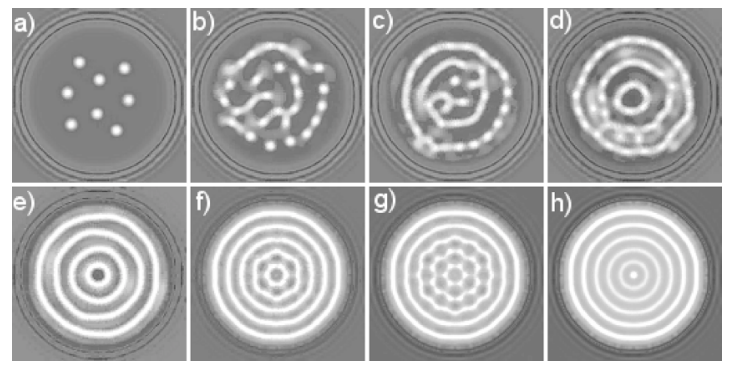

FIG. 16. The intensity field profile changes when the input field is slowly increased from a value $\left(E_{I}=0.66\right)$ slightly lower than the threshold $A$ to a value $\left(E_{I}=2.5\right)$ well beyond threshold $B$. The system displays the patterns whose maxima correspond to the circles in Fig. 15. Patterns in (b), (c), (d) are dynamical, all the others are stationary. Gray scale ranges from white (intense fields) to black (low fields).

well known that in fluid dynamics convective rolls connect orthogonally to the boundary [48]. In our case we performed a slow forward scan of the input field intensity with a constant cavity detuning $(\eta=0)$ and studied the pattern variety observed above the MI threshold. In Fig. 16 we show the evolution of spatial structures for input field values ranging from 0.66 to 2.5 .

We start from an initial condition [Fig. 16(a)] corresponding to isolated CSs sitting on the homogeneous background; by increasing the input intensity CSs are no longer stable and dynamical transverse filaments appear [Fig. 16(b)], at times breaking up in spots then reconnecting again, but invariably the outermost structures tend to assume the circular symmetry of the boundary [Fig. 16(c)]. Upon further increase, the filaments tend to stabilize in circular rings which attain a prefect regularity for still higher fields. The final targetlike structures show the tendency of the patterns to avoid contact with the boundaries, as opposed to the hydrodynamical case.

This branch of patterns is indefinitely extended, in the sense that the system's boundaries cause the disappearance of the upper MI threshold $B$ in Fig. 15. Beyond the plane wave (PW) input intensity, corresponding to threshold $B$, the emitted field profile should rather soon fall back to the homogeneous solution (it has a subcritical character as the lower one); on the contrary we found that by continuously increasing the input intensity well above this threshold the emitted field preserves a circular symmetry. In order to confirm that the occurrence and persistence of target patterns can be ascribed to the role of the boundaries, we ran simulations for $E_{I}=3.0$, a value far beyond the threshold $B$ in Fig. 15, where a target pattern is realized [Fig. 17(a)]. Then the system was integrated again after increasing the spatial step, which amounts to simulate a sample of larger diameter, so that the effect of the sample borders on the field spatial structure is diminished. Results are shown in Fig. 17(b): the central region of the sample shows a flat profile, now corresponding to the value of the homogeneous solution. The persistence of a weaker, circular motif on the border ensures that the diffractive modulations are still present, i.e., patterns are not artificially canceled by a grid too coarse to accommodate transverse modulations. This confirms that the ringlike patterns are intrinsic structures, stabilized beyond the MI 

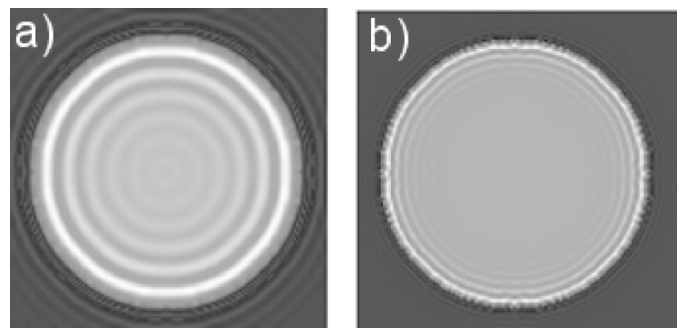

FIG. 17. The field intensity profile for $E_{I}=3.0$ as marked in Fig. 15. We expect an unbounded device to be homogeneous here because the system is way above the higher MI threshold $B$. In (a) we see that a pattern is still present, though, as a result of a boundaryinduced stabilization (see text). The spatial step size is 0.5. (b) The spatial step size has been increased to 1.2 , thus simulating a broader device. The target pattern has disappeared and the profile in the emission circle is almost homogeneous, save for a few diffraction rings at the sample border.

threshold $B$ by the sample boundaries, and that when this constraint is removed the system realizes the homogenous solution as the PW analysis predicts.

The second step in our analysis consisted in introducing the cavity resonance gradient and reproducing the pattern variety under broken azimuthal symmetry. It is worthwhile here to recall how our previous theoretical and experimental investigations evidenced the onset of two separated regions for patterns and homogeneous profile. According to our results, in an appropriate range of values of the holding beam intensity, the reflected field shows on the left a dynamical pattern, whose intensity peaks irregularly oscillate in time, and a homogeneous region on the right [see Ref. [39], Fig. $3(\mathrm{~b})$ ]. Although depending on $\theta_{1}$, the location of the boundary between the two does not coincide with the line $x$ $=\theta_{1} / \eta$ [see Eq. (4)], where the cavity is resonant with the input field; actually, it is determined by the threshold of the modulational instability causing the formation of patterns; as it is well known, this threshold depends on the cavity detuning (among other parameters, such as the input field intensity, see below), hence, when it changes across the sample, one finds the boundary in terms of the locus of the local MI thresholds. Note that it reproduces a nearly straight line when the input field is almost a plane wave. Our interpretation is supported by an analytical evaluation of the MI threshold for an unbounded system, with PW injection [see Ref. [39], Fig. 3(a)] where the predicted threshold matches the numerical evidences, and we also provide an indication about the sample region where CSs are expected to be stable.

\section{Comparison with the experiment}

Since in the experiment a shift of the boundary was observed by changing either the input field frequency or its intensity, we validated this evidence in terms of the drift of the MI threshold with those two quantities. Simulations fully confirm this behavior as seen in Figs. 18 and 19. In Fig. 20 we show an interpretation of the slight boundary curvature observed at times in the experiment as due to the Gaussian shape of the input field whose isointensity lines are circles:

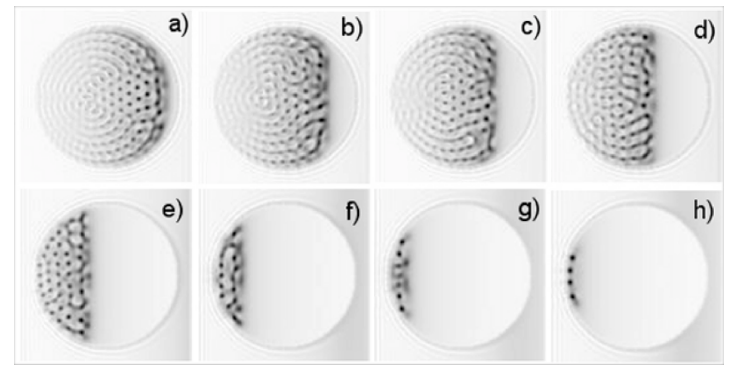

FIG. 18. Sample with high cavity resonance gradient. Field intensity profile (gray scale) for decreasing values (from $a$ to $h$ ) of the cavity detuning $\theta$. This matches the experimental observed shift of the boundary upon decreasing the injected wavelength (see Fig. 5).

for weaker local intensities the threshold is pushed towards lower cavity detunings (as hinted by Fig. 19). As it turns out, a beam waist around the size of the sample diameter makes the HB departures from plane wave irrelevant to all practical effects.

Another agreement between experiment and theory here is the reduction of the structure size when the cavity detuning becomes smaller, as a consequence of the increase of the modulational instability's critical wave vector $K_{C}$ for decreasing cavity detunings, as predicted in the past [49]. By injecting higher and higher intensities, the boundary moves rightwards and the patterns invade the whole device's section. As in the previous case with constant detuning, the patterns (filaments, occasionally breaking up in spots) are dynamical, and they continuously evolve in time. Eventually, when the pattern occupies the whole section of the device it undergoes a process similar to that observed in the previous case, and the field shows the occurrence of the target patterns with unaffected circular symmetry [same as Fig. 16(h)].

The last part of our investigations was devoted to a characterization of the CSs properties, as evidenced by the experiments. The peculiarities of the CSs observed in this par-

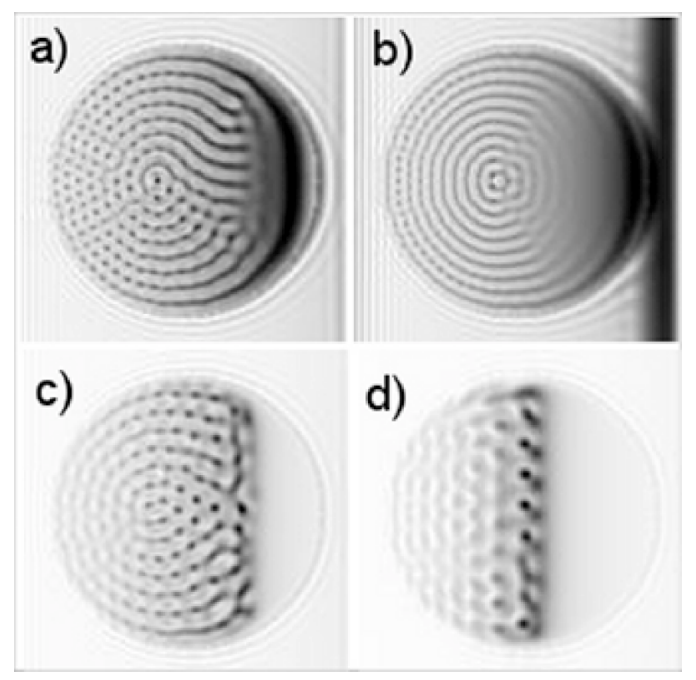

FIG. 19. Sample with high cavity resonance gradient. Field intensity profile (gray scale) for decreasing (from $a$ to $d$ ) values of the input intensity. This matches the experimental observed border shifting upon decreasing the injected power (see Fig. 6). 

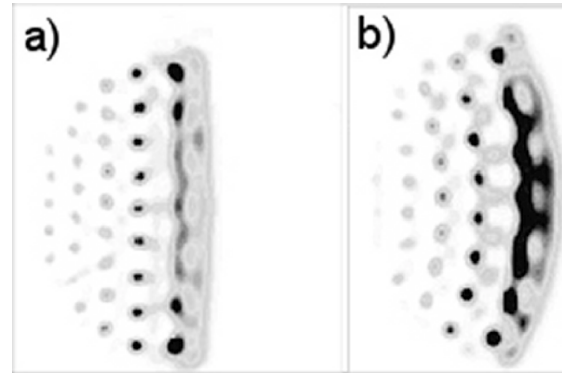

FIG. 20. The effect of the holding beam shape on the emitted field profile: from (a) to (b) the HB waist has been halved.

ticular experiment, that in our opinion needed an interpretation and/or an at least partially quantitative comparison, were (a) the stabilization of CSs in determined locations of the sample section, which was spontaneously achieved just in the sample with low resonance gradient, while in the sample with high transverse gradient it could be obtained only by making use of an eccentric, narrower holding beam; and (b) the possibility of controlling the stationary CSs by adding a further Gaussian external beam.

A general theoretical treatment of the CS dynamical response in presence of external perturbations, in particular of those amounting to a parameter gradient in the spatial domain (be it phase, intensity, or detuning), was reported in Refs. $[17,46]$. The main result was the prediction of a linear dependence of the CS drift speed to the gradient of the spatially varying quantity, as shown by Eq. (6.15) of Ref. [46], when the gradient could be treated perturbatively. Although the detuning gradient in the investigated sample cannot be treated perturbatively, we could nevertheless expect the detuning gradient to act as a sort of Aristotelian force where the velocity is vectorially proportional to the force itself. We thus started to perform simulations for the high-gradient sample, by estimating a resonance (and thus detuning) drift of about five scaled units in our parameter $\theta$ for a resonator with an average of $0.5 \%$ transmittivity.

The CSs were excited by adding a narrow pulse to the holding external field with its same phase, for a short time, as reported in Ref. [15], and they immediately behaved as expected, sliding leftwards and following the gradient lines. As it turns out, the CS slides directly into the patterned domain, melting with the structures and ceasing to exist as an individual entity. We tried to reproduce the experimental technique by means of which it was possible to compensate the speed induced by the force associated to detuning gradient with an equal and opposite force associated to the intensity gradient intrinsic to the Gaussian profile of the holding beam.

Yet, we could not cause a CS to stop by merely acting on the sole holding beam, therefore we added a second Gaussian beam [hereinafter control beam (CB)], with a lower intensity (about one order of magnitude) than the holding beam and a considerably narrower waist (about $1 / 25$ ). This accomplished the double requirement of providing a sensible intensity gradient (variation in space) and a negligible local perturbation (intensity magnitude) which did not affect the CSs stability. A typical three-dimensional (3D) intensity portrait
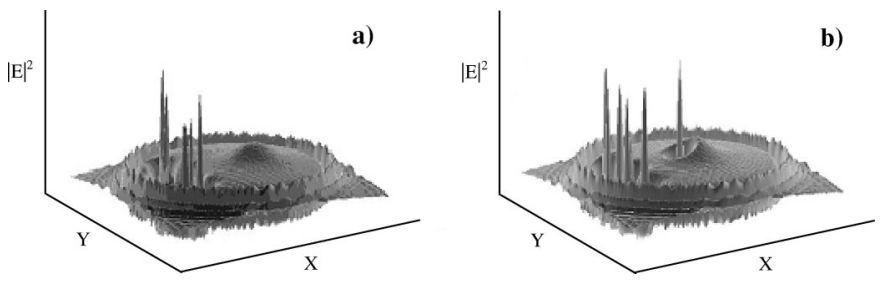

FIG. 21. A 3D surface plot of the transverse field intensity showing a system state similar to that shown in 2D in Fig. 18(a). (a) The small hump appearing in the homogeneous emission domain, to the right of the patterned region, shows the local-field increase due to the additional beam $\mathrm{CB}$. The $\mathrm{CB}$ is weak enough as not to trigger modulational instabilities at the location where itis centered. (b) Here the system exhibits the profile at regime after a suitable pulse has locally excited a CS at the far end of the sample. The CS sled towards the patterned region due to the detuning gradient and has been eventually stopped by the countergradient (in intensity) applied by the CB. The plotted profile is thus a stationary one. Note that the CS is stopped on the left of the CS maximum, because that is where the detuning and intensity gradients are opposite (antiparallel); to its right the two would be parallel.

for this double Gaussian injection is shown in Fig. 21(a). When a CS is now switched on at a location lying at the left of the $\mathrm{CB}$ axis, it starts sliding leftwards, crosses the $\mathrm{CB}$ maximum and starts feeling the opposite gradient force. It then slows down, and remains trapped in a precise location, where the two forces cancel. The equilibrium regime is depicted in Fig. 21(b).

The second sample which was experimentally investigated exhibited a gradient one order of magnitude weaker, and in those conditions CSs were observed sliding towards certain location where they could stand still.

Based on our previous experience of the role of the roughness in the detuning profile (due to the layer jumps intrinsic to the DBR epitaxial deposition [34]) we interpreted the existence of equilibrium positions as the results of local sizeable variations in the detuning so that, if we may introduce a pictorial image, the CS can be fancied as a rock rolling downhill that can be stopped by a hump (or a dip) in the terrain, provided it is sufficiently elevated (or hollow). The milder the slope, the easier is to find a land scar capable to accomplish the trapping. We thus performed the new streams of simulations adding the stochastic realizations of the detuning profile described above [see Fig. 14].

Indeed, as shown in Fig. 22, the CS moves to the left with a velocity of about $550 \mathrm{~m} / \mathrm{s}$, reaches a location where it gets trapped and there it will sit still forever. It must be noted that

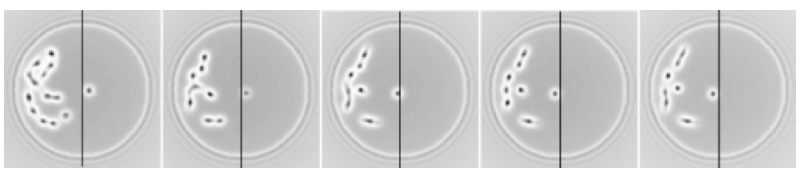

FIG. 22. The CS slides leftwards and is trapped in a roughnessdetermined location. As commented in the text, the patterns in the left domain are not stationary, but continuously evolve in time. A vertical diameter has been added to make the movement more evident. The last frame represents a stationary configuration, whose 3D profile appears in Fig. 23. 


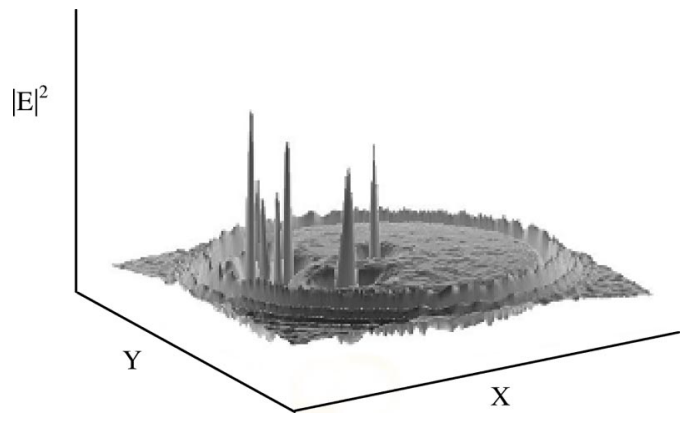

FIG. 23. The stationary profile of the field intensity after a CS (originally excited around the sample center) has slid along the detuning gradient and has been stopped by the sample roughness (see text). Compare the relative roughness in the detuning profile (Fig. 14) and the smoothness in the homogenous region of the emitted field in the background of the CS. The arrow evidences the CS. Despite its vicinity to the pattern boundary the CS can still be addressed independently.

the roughness-induced equilibrium locations in principle limit the continuum of positions where a CS can be encoded to a countable set, but, on the other hand, they appear in our simulations to be interspersed densely enough throughout the sample's cross section, as to ensure a satisfactory density of equilibrium positions. It must be kept in mind that there exist interaction distances between CSs $[15,17,28,46]$, and the CSs spatial encoding density seems to be more stringently limited by this factor. Also, in absence of the roughness trapping, CS would slide undisturbed and one would need to largely reduce the diametral resonance gradient in the sample, with a consequent increase in sample costs and growth times.

Figure 23 shows a 3D plot of the intensity profile, where the reader can appreciate the absence of strong perturbations induced by the cavity detuning roughness, whose main effect actually resides in the dynamical action of the local fluctuations on the Cs motion, rather than on the local intracavity field. The background intensity is almost as smooth as in Fig. 21(a).

\section{CSs switch-on}

Finally we devoted a series of simulations to the evaluation of the dynamics of the CSs switch-on process, in order to reproduce the experimental results concerning CS build-up times. We start our study from the PW analysis [see Fig. 15], in a parameter range where the CSs branch coexists with the lower homogeneous branch. By injecting a narrow WB into the cavity for a short interval of time we can access the CSs branch and create a soliton. Practically, when the holding beam has an amplitude $\left|E_{I}\right|=0.75$ in our scaled units [see Fig. 15], we add a WB with a Gaussian shape centered at an arbitrary position. The duration of the injection can be optimized to a minimum value, below which the CS cannot be created. Nevertheless, this parameter is not significant from an experimental viewpoint, because the experimental time resolution in analyzing the injection process is too coarse (experimentally the duration of the injection is around $100 \mathrm{~ns}$, and cannot be significantly reduced). We therefore

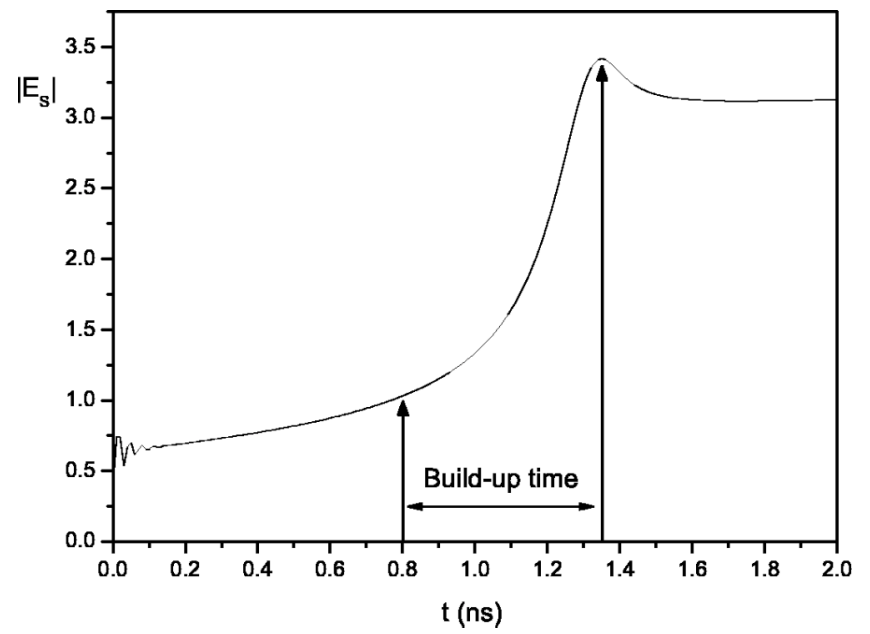

FIG. 24. Modulus of the intracavity field at the CS peak as a function of time, during injection of a Gaussian pulse of $15 \mu \mathrm{m}$ width (numerical simulation).

fixed the WB waist to $\sigma_{W B}=15 \mu \mathrm{m}$, the duration of the injection to $20 \mathrm{~ns}$, and the WB amplitude $\left|E_{W B}\right|=0.5$ in our scaled units. In Fig. 24 we report the amplitude of the intracavity field at the CS center during the first 2 ns of the injection. After a delay time (of about $0.8 \mathrm{~ns}$ ), the CS switches on with a build-up time of roughly $550 \mathrm{ps}$. As for the CS build-up time, the agreement with the experiment is excellent (experimental value: $570 \pm 50 \mathrm{ps}$ ). Next we introduced a phase mismatch between the two beams. As the phase of the WB is varied with respect to the holding beam, ranging from 0 to \pm 20 , we note that the build-up time remains unchanged.

The second step is to evaluate the WB power necessary to switch on a CS, in order to compare it with the experimental findings. In our theoretical approach we are used to work with scaled quantities, therefore it is very difficult to evaluate the real physical quantities. So we decided not to compare the absolute powers, but the ratio between the WB power $\left(P_{W B}\right)$ and the HB power $\left(P_{H B}\right)$. Experimentally [39], typical values are $P_{W B}=50 \mu \mathrm{W}$ and $P_{H B}=8 \mathrm{~mW}$, so that $P_{W B} / P_{H B}$ $=6.25 \times 10^{-3}$. Numerically, we have to evaluate $P_{W B} / P_{H B}$ $=\left|E_{W B}\right|^{2} \pi \sigma_{W B}^{2} /\left|E_{I}\right|^{2} A$, where $A$ is the area of the sample. After substituting the value we currently used, we obtain $P_{W B} / P_{H B}=1.40 \times 10^{-2}$, with a good agreement with the experimental value. The agreement becomes even better $\left(P_{W B} / P_{H B}=4.44 \times 10^{-3}\right)$, if we inject a Gaussian (instead of a PW) holding beam with a waist $\sigma_{H B}=150 \mu \mathrm{m}$, as in the experiment.

\section{CONCLUSIONS AND DEVELOPMENTS}

By comparing theoretical models and the extensive simulations based thereupon to a number of experimental evidences, we have provided a rather complete analysis of the pattern features and CSs properties, as observed in the field profile emitted by a broad-area VCSEL amplifier. The fundamental properties of CSs have been assessed and the crucial measurements concerning their addressing, drift and pinning have been validated by theoretical interpretations. In particu- 
lar we have shown that, despite an intrinsic cavity resonance gradient and residual layer imperfections (due to the epitaxial growth process), CSs still exhibit independence and mobility, and thus remain viable for dynamical and controlled optical information encoding and processing. Both theoretical and experimental activities are in progress to realize schemes where such basic features are applied to relevant all-optical applications such as buffer register, serial-parallel conversion, and array reconfiguration. The agreement between simulations and observations is excellent, even quantitatively on some issues such as the CS switch-on times and powers requirement for the injection beams.

\section{ACKNOWLEDGMENTS}

The INLN group acknowledges ACI photonique "Contrôle et manipulation de solitons de cavité dans les systèmes optiques auto-organisés." The groups in Bari and Como acknowledge the PRIN project "Formazione e controllo di solitoni di cavitá in microrisonatori a semiconduttore" of the Italian Ministry of University and Research, and the European Network VISTA (VCSELs for Information Society Technology Applications).
[1] H. Haken, Synergetics, and Introduction (Springer-Verlag, Berlin, 1997).

[2] G. Nicolis, Introduction to Nonlinear Science (Cambridge University Press, Cambridge, 1995).

[3] F. T. Arecchi, Physica D 51, 450 (1991).

[4] W. J. Firth, in Self-Organization in Optical Systems and Applications in Information Technology, edited by M. A. Vorontsov and W. B. Miller (Springer-Verlag, Berlin, 1995), p. 69.

[5] L. A. Lugiato, M. Brambilla, and A. Gatti, in Advances in Atomic, Molecular and Optical Physics, edited by B. Bederson and H. Walters (Academic, New York, 1998), vol. 40, p. 229.

[6] G. I. Stegeman and M. Segev, Science 286, 1518 (1999).

[7] O. Thual and S. Fauve, J. Phys. (France) 49, 1829 (1988).

[8] G. Dewel, P. Borckmans, A. de Wit, B. Rudovics, J. J. Perrand, E. Dulos, J. Boissonade, and P. de Kepper, Physica A 213, 181 (1995)

[9] P. Coullet, C. Riera, and C. Tresser, Phys. Rev. Lett. 84, 3069 (2000).

[10] D. W. McLaughlin, J. V. Moloney, and A. C. Newell, Phys. Rev. Lett. 51, 75 (1983).

[11] N. N. Rosanov and G. V. Khodova, Opt. Spectrosc. 65, 449 (1988).

[12] G. S. McDonald and W. J. Firth, J. Opt. Soc. Am. B 7, 1328 (1990).

[13] M. Tlidi, P. Mandel, and R. Lefever, Phys. Rev. Lett. 73, 640 (1994).

[14] W. J. Firth and A. J. Scroggie, Phys. Rev. Lett. 76, 1623 (1996).

[15] M. Brambilla, L. A. Lugiato, and M. Stefani, Europhys. Lett. 34, 109 (1996)

[16] N. N. Rosanov, in Progress in Optics, edited by E. Wolf (North Holland, Amsterdam, 1996), Vol 35, p. 1.

[17] W. J. Firth and G. K. Harkness, Asian J. Phys. 7, 665 (1998).

[18] V. Y. Bazhenov, V. B. Taranenko, and M. V. Vasnetsov, Proc. SPIE 1806, 14 (1993).

[19] M. Saffman, D. Montgomery, and D. Z. Anderson, Opt. Lett. 19, 518 (1994).

[20] C. O. Weiss, M. Vaupel, K. Staliunas, G. Slekys, and V. B. Taranenko, Appl. Phys. B: Lasers Opt. B68, 151 (1999).

[21] A. Schreiber, B. Thuering, M. Kreuzer, and T. Tschudi, Opt. Commun. 136, 415 (1997).

[22] P. L. Ramazza, S. Ducci, S. Boccaletti, and F. T. Arecchi, J. Opt. B: Quantum Semiclassical Opt. 2, 399 (2000).
[23] B. Schapers, M. Feldmann, T. Ackemann, and W. Lange, Phys. Rev. Lett. 85, 748 (2000).

[24] Z. Chen and K. McCarthy, Opt. Lett. 27, 2019 (2002).

[25] J. Petter, J. Schroeder, D. Traeger, and C. Denz, Opt. Lett. 28, 438 (2003).

[26] M. Brambilla, L. A. Lugiato, F. Prati, L. Spinelli, and W. J. Firth, Phys. Rev. Lett. 79, 2042 (1997).

[27] D. Michaelis, U. Peschel, and F. Lederer, Phys. Rev. A 56, R3366 (1997).

[28] L. Spinelli, G. Tissoni, M. Brambilla, F. Prati, and L. A. Lugiato, Phys. Rev. A 58, 2542 (1998).

[29] G. Tissoni, L. Spinelli, M. Brambilla, T. Maggipinto, I. M. Perrini, and L. A. Lugiato, J. Opt. Soc. Am. B 16, 2083 (1999a).

[30] G. Tissoni, L. Spinelli, M. Brambilla, T. Maggipinto, I. M. Perrini, and L. A. Lugiato, J. Opt. Soc. Am. B 16, 2095 (1999b).

[31] L. Spinelli, G. Tissoni, M. Tarenghi, and M. Brambilla, Eur. Phys. J. D 15, 257 (2001).

[32] J. Scheuer and M. Orenstein, Science 285, 230 (1999).

[33] V. B. Taranenko, I. Ganne, R. J. Kuszelewicz, and C. O. Weiss, Phys. Rev. A 61, 063818 (2000).

[34] R. Kuszelewicz, I. Ganne, I. Sagnes, G. Slekys, and M. Brambilla, Phys. Rev. Lett. 84, 6006 (2000).

[35] T. Ackemann, S. Barland, M. Cara, S. Balle, J. R. Tredicce, R. Jaeger, M. Grabherr, M. Miller, and K. J. Ebeling, J. Opt. B: Quantum Semiclassical Opt. 2, 406 (2000).

[36] T. Ackemann, S. Barland, J. R. Tredicce, M. Cara, S. Balle, R. Jaeger, M. Grabherr, M. Miller, and K. J. Ebeling, Opt. Lett. 25, 814 (2000).

[37] V. B. Taranenko, I. Ganne, R. Kuszelewicz, and C. O. Weiss, Appl. Phys. B: Lasers Opt. B72, 377 (2001).

[38] I. Ganne, G. Slekys, I. Sagnes, and R. Kuszelewicz, Phys. Rev. E 66, 066613 (2002).

[39] S. Barland, et al., Nature (London) 419, 699 (2002).

[40] L. A. Lugiato, IEEE J. Quantum Electron. 39, 193 (2003).

[41] M. Grabherr, R. Jaeger, M. Miller, C. Thalmaier, J. Herlein, and K. J. Ebeling, IEEE Photonics Technol. Lett. 10, 1061 (1998).

[42] S. Barland, F. Marino, M. Giudici, J. Tredicce, and S. Balle, Appl. Phys. Lett. 83, 2303 (2003).

[43] T. Knoedl, M. Miller, and R. Jaeger (private communication).

[44] H. Haug and S. W. Koch, Quantum Theory of the Optical and 
Electronic Properties of Semiconductors, 2nd ed. (World Scientific, Singapore, 1993).

[45] C. H. Henry, IEEE J. Quantum Electron. 18, 259 (1982).

[46] T. Maggipinto, M. Brambilla, G. K. Harkness, and W. J. Firth, Phys. Rev. E 62, 8726 (2000).
[47] T. Maggipinto, M. Brambilla, and W. J. Firth, IEEE J. Quantum Electron. 39, 206 (2003).

[48] P. Manneville, Dissipative Structure and Weak Turbulence (Academic, San Diego, 1990).

[49] L. A. Lugiato and C. Oldano, Phys. Rev. A 37, 3896 (1988). 